\title{
Malignant Transformation of Labial Lichen Planus: A Rare Case Report
}

\author{
Raouâa Belkacem Chebil1*, Moussaoui Eya¹, Abdelfattah Zakhama², Habib Hamdi1 \\ ${ }^{1}$ Oral medicine oral surgery department, Monastir School of dental medicine, Monastir University, 5000 Monastir, Tunisia \\ ${ }^{2}$ Cytology and pathological anatomy department, Monastir School of medicine, 5000 Monastir, Tunisia
}

\section{*Corresponding Author}

Raouâa Belkacem Chebil

\author{
Article History \\ Received: 15.07.2019 \\ Accepted: 25.07 .2019 \\ Published: 30.07 .2019
}

\begin{abstract}
Oral lichen planus (OLP) is a T-cell-mediated chronic inflammatory disease of the oral mucosa. It has been considered by many authors as a premalignant condition. In some reported cases, there is lack of clinical and histological data leading to a wrong estimation of its malignant potential. We report the case of a 74-year-old woman who had been diagnosed with erosive OLP extending over gingiva, labial and buccal mucosa with absence of known exogenous carcinogens. Six years after, verrucous lesion appeared on labial lesion. Topical antifungal treatment was initially prescribed to treat a deep candida infection. Then, a biopsy confirmed the diagnosis of squamous cell carcinoma. Radiological examination confirmed absence of lymph nodes or metastatic lesion. Total surgical excision was performed with respect of carcinologic resection margins. Neither local recurrence nor second primary tumor was noted during 5 years of follow-up. The present case supports the view that OLP may undergo malignant transformation, and that this does not require exogenous carcinogens. It highlights the necessity of correct record of clinical, histological and treatment details to make possible and reliable the diagnosis of OLP malignant transformation. There is still a need of close follow-up of OLP and lichenoid lesion patients especially when having associated other carcinogenic risk factors.
\end{abstract}

Keywords: Oral lichen planus, candida infection, lichenoid

\section{INTRODUCTION}

Oral lichen planus (OLP) is a T-cell-mediated chronic inflammatory disease of the oral mucosa. Its possible malignant potential remains a serious debate issue. The World Health Organization defined the pre-malignant condition as "a generalized condition associated with a significantly increased risk of cancer" and includes OLP in this classification [1]. The major topic of discussion is whether to consider OLP as an intrinsically premalignant condition or simply as a facilitator of the action of the carcinogenic agents Such as smoking, alcoholism, nutritional errors, poor hygiene, immunosuppression and oncogenic viruses.

The reported malignant transformation rate is often higher for erosive and atrophic forms and depends on the studied population and the diagnostic criteria used. In many of the reported studies, there is insufficient clinical and histopathological data to confirm the initial diagnosis of OLP. Krutchkoff et al. [2] established criteria for acceptance of a reported case of malignant transformation in OLP. OLP, OLL and proliferative verrucous leukoplakia (PVL) may present clinical and microscopic similarities making more difficult the appreciation of OLP malignant potential.

The study of the clinical behavior of malignant transforming oral lichen planus has revealed that patients with OSCC developed on a preexisting OLP lesions showed a higher rate of tumor recurrence when compared to those with primary OSCC. Thus, some authors recommend a more radical treatment of OSCC arisen in OLP and a strict post-operative follow-up period [3].

Here, we report the case of a patient with a lesion previously diagnosed as oral lichen planus, who developed squamous cell carcinoma in the same location, in the absence of known exposure to exogenous carcinogens.

\section{CASE REPORT}

A 74 years-old woman was referred in 2006 to the department of oral medicine and oral surgery in Monastir dental university clinic for evaluation of buccal, labial and gingival erosive lesions. Her medical history revealed that she had type 2 diabetes with high

Copyright @ 2019: This is an open-access article distributed under the terms of the Creative Commons Attribution license which permits unrestricted use, distribution, and reproduction in any medium for non commercial use (NonCommercial, or CC-BY-NC) provided the original author and source are credited. 
blood pressure and dyslipidemia. She had had an acute myocardial infarction 2 years before, which was treated by percutaneous coronary angioplasty. She was taking the following medications: TAHOR®, CORVASAL $®$, HYPOTEN®, LASILIX®, ASPEGIC® and GLUCOPHAGE $®$. She was a non-smoker and did not consume alcohol. At time of referral, the lesions were bilateral and erosive extending over gingiva, labial and buccal mucosa. Generalized gingival inflammation was noted with typical white striae (figure 1). Lesions were clinically diagnosed as erosive oral lichen planus. A biopsy was taken from lower labial mucosa near to the right labial commissure. The diagnosis of erosive lichen planus was thus confirmed (figure2) and treatment was instaured with topical steroids as mouthwash three times daily (solupred®). At next appointment, the patient showed symptom improvement but examination revealed a persistent erosive lesion surrounded by keratotic plaque close to the right labial commissure (figure $1 \mathrm{~B}$ ). A second biopsy was taken from the same site and histopathological diagnosis was oral lichen planus deeply infected with candida without signs of dysplasia. Topical antifungal ointment transformed the heterogeneous lesion into an homogenous keratotic plaque. Patient was seen every four months during 6 years with absence of erosive area. When controlling the lesions in november 2011, there were recurrence of erosion in the same area which became verrucous after 4 months. The same topical antifungal ointment (DAKTARIN®) was prescribed twice a day and the patient was seen after 1 month for total excision of the labial lesion to exclude malignant transformation. Excisional biopsy performed in June 2012 revealed a well differentiated squamous cell carcinoma with non-free margins (figure 3). There were no palpable enlarged cervical lymph nodes. Computed tomography confirmed absence of lymph nodes or metastatic lesion. A Fourth intervention with total excision of the granular keratotic area (figure 4) was performed with respect of $5 \mathrm{~mm}$ of resection margins (figures 5,6).No local recurrence nor second primary tumor was noted during 5 years of follow-up (figure7).
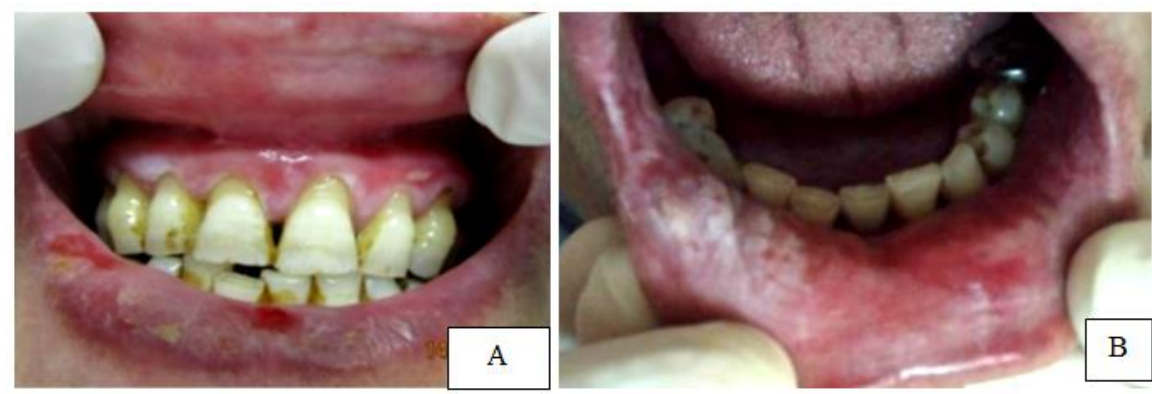

Fig-1: A: at initial examination, generalized gingival inflammation with typical keratotic areas. B: After 4 months: erosive mamelonated labial lesion surrounded by keratotic plaque close to the right labial commissure
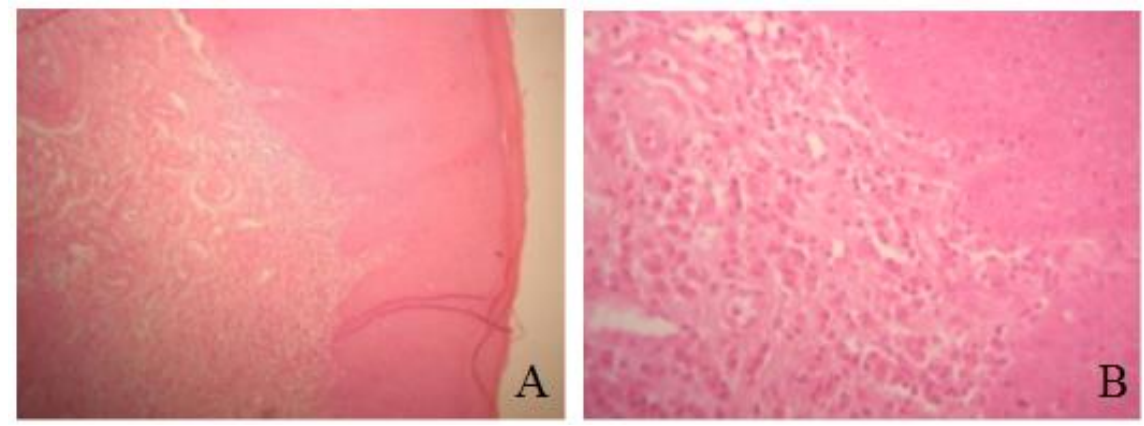

Fig-2: First biopsy showing erosive oral lichen planus without signs of dysplasia. A: Hematoxylin-eosin, 10x; B: Hematoxylin-eosin, 40x.

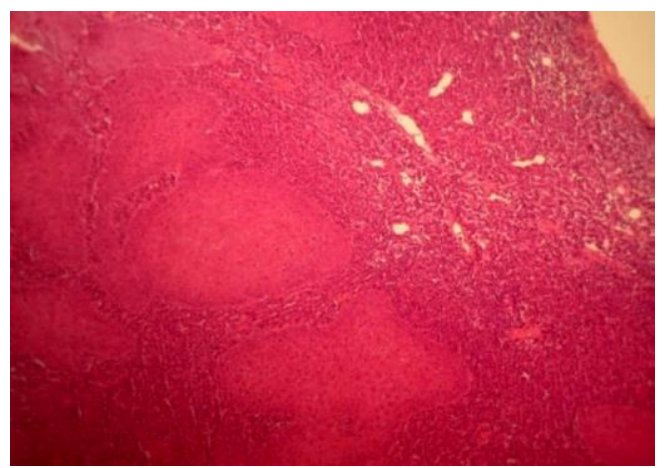

Fig-3: Biopsy of the squamous cell carcinoma lesion, showing well-differentiated squamous cells. (Hematoxylin-eosin, 40x) 


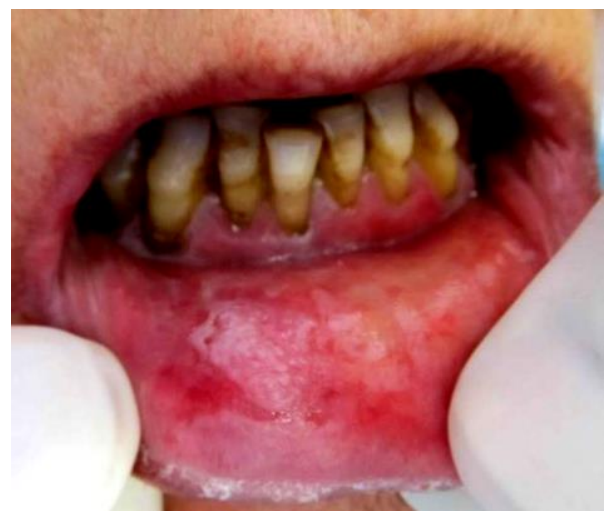

Fig-4: Labial lesion 7 weeks after squamous cell carcinoma confirmation: erythematous lesion associated with granular keratosis

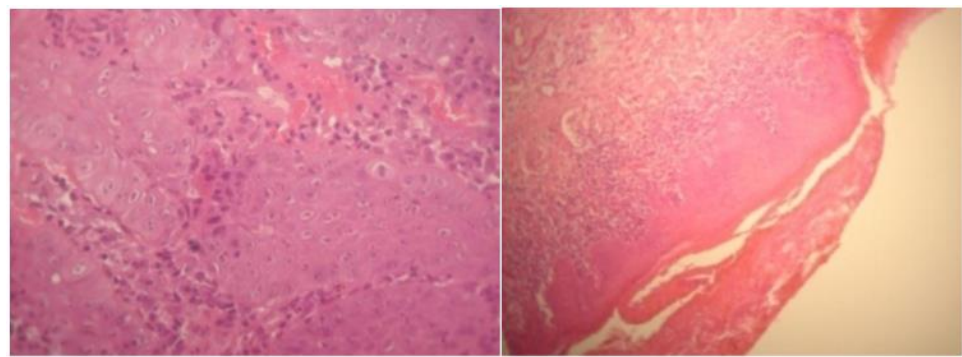

Fig-5 and 6: Histological examination of the squamous cell carcinoma lesion, showing well-differentiated squamous cells. Peripheral limits showed OLP without dysplasia: right side: Hematoxylin-eosin, 10x, left side: B: Hematoxylin-eosin, 100x

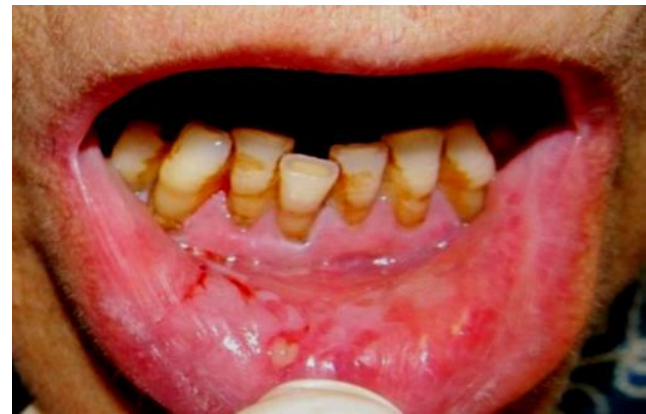

Fig-7: Inner surface of the lower lip: clinical aspect 12 days after complete surgical excision

\section{DISCUSSION}

Although many authors suggested that oral lichen planus is a premalignant condition, other authors have disputed this view. Krutchkoff et al. [2] in a review of 223 cases published between 1950 and 1976, established strict criteria for acceptance of a reported case of oral lichen planus undergoing malignant transformation (table 1). All of these criteria were fulfilled for our patient. Original diagnosis was based on modified WHO criteria proposed by Van der Meij and Van der Waal. The patient was followed for six years with correct record of clinical and treatment details. Labial lesion was twice biopsied confirming absence of dysplasia in the same area where malignant transformation was noted six years after.

Table-1: criteria of acceptance of malignant transformation of Oral Lichen Planus [2]

\begin{tabular}{|l|l|}
\hline Original Diagnosis & The diagnosis would include at least the last two of the following four recognized \\
& features: \\
& (1) Hyper- or parakeratosis \\
& (2) Saw-toothed retepegs \\
& (3) Superficial infiltrate of lymphocytes \\
& (4) Basal cell liquefaction. \\
\hline History and Follow-up & reported transformations should have had proper follow-up (a minimum of 2 years) with \\
& all changes in \\
\hline Details of Treatment and Tobacco & clinical features properly recorded. \\
Exposure & All forms of intercurrent treatment and/or tobacco habits should also have been properly \\
\hline
\end{tabular}


Giuliani et al. conducted a systematic review to determine the malignant transformation rate of OLP and oral lichenoid lesion and their risk factors [4].

He found an overall transformation rate of $1.40 \%$ (1.37\% for OLP and $2.43 \%$ for OLL) and an annual transformation rate of $0.20 \%$. He concluded also that female gender, erosive forms and tongue site should be considered as risk factors for OLP transformation. The higher transformation rate of OLL may be related to strict application of OLP diagnostic criteria proposed by van der Meij and van der Waal in which the presence of epithelial dysplasia precludes a diagnosis of OLP.

A recent meta-analysis reported that $1.1 \%$ of OLP lesions progress into OSCC with a higher incidence in smokers, alcohol users, and in those infected with hepatitis $C$ virus [5]. The available data did not allow performing a statistical analysis on the impact of these classic risk factors (smoking and alcohol) in the determinism of malignant transformation. Studies on rats have shown that candida is able to produce carcinogens such as N-Nitrosamine which have a synergistic effect when associated to these classic risk factors (smoking and alcohol) that can predispose to neoplastic evolution [4].

In this reported case, malignant transformation was noted on OLP lesion diagnosed according to van der Meij and van der Waal criteria which makes this observation very rare. Three risk factors may be considered the female gender, erosive feature and candida infection. Persistent erosion was noticed on the inner surface of the lower lip behaving suspiciously which indicated biopsy to rule out neoplastic change. Mycelium filaments observed on microscopic examination confirmed chronic and deeply infected lesion which might play a role on malignant transformation.

The major obstacle to assuring the validity of studies investigating OLP potential for undergoing malignant transformation is the lack of reliable diagnostic criteria making possible the differential diagnosis between OLP and OLL and their differentiation from other oral diseases especially from proliferative verrucous leukoplakia (PVL). This latter lesion frequently shows clinical and microscopic features similar to those found in OLP particularly in early stages when verrucous change and cellular atypia are not yet present. Clinically PVL can appear as hyperkeratotic plaques, erythematous atrophic regions, and ulceration. On histopathological examination, it shows hyperkeratosis associated with lichenoid infiltrate resembling OLP. However, the presence of verrucous epithelial architectural change and varying degrees of cellular atypia distinguishes PVL from OLP [6]. The absence of verrucous and dysplastic changes in our patient confirmed the diagnosis of OLP and excluded PVL.

Recently a position paper of the American Academy of Oral and Maxillofacial Pathology proposed a new set of clinical and pathological criteria (table 2). They required that absence of a verrucous epithelial architecture is necessary before rendering a diagnosis of OLP which was not recommended in previous classifications criteria [7].

Table-2: American Academy of Oral and Maxillofacial Pathology diagnostic criteria of OLP [7]

Clinical criteria

- Multifocal symmetric distribution

- White and red lesions exhibiting one or more of the following forms

$\checkmark$ Reticular/ papular

$\checkmark$ Atrophic (erythematous)

$\checkmark$ Erosive (ulcerative)

$\checkmark$ Plaque

$\checkmark$ Bullous

- Lesions are not localized exclusively to the sites of smokeless tobacco placement

- Lesions are not localized exclusively adjacent to and in contact with dental restorations

- Lesion onset does not correlate with the start of a medication

- Lesion onset does not correlate with the use of cinnamon-containing products

Histopathologic criteria

- Band-like or patchy, predominantly lymphocytic infiltrate in the lamina propria confined to the epithelium-lamina propria interface

- Basal cell liquefactive degeneration

- Lymphocytic exocytosis

- Absence of epithelial dysplasia

- Absence of verrucous epithelial architectural change

The pathogenesis of OLP and the mechanism for malignant transformation are still unclear. The prevailing theory proposes that the neoplastic process is based on increased proliferation of the basal-layer cells under the influence of mediators released from the inflammatory infiltrate that activate different pathways and can lead to tumor development [8]. 
Recently, a significant amount of studies aimed at identifying reliable biomarkers that can predict the malignant transformation of OLP lesions. Alterations in the expression of numerous biomarkers showed promising results, including modulators of apoptosis (p53, MCL-1), cell cycle regulators (BMI1, p16), tissue remodeling factors (MMPs), and inflammation-related factors (TNF-a, IL-6, and COX-2) [9].

Mignogna et al. [10] studied the clinical behavior of malignant transforming oral lichen planus from follow-up data of 21 patients. He found that OLP features in patients developing OSCC did not show clinical differences from other OLP patients. However, OLP-related OSCC behavior was marked by a worse prognosis with a tendency in developing second primary metachronous tumors of the oral mucosa and nodal metastases. For this reason, he recommended a more radical treatment of OSCC arisen in OLP and a strict follow-up with oral and neck examination every 2 months for $6 \pm 9$ months in the postoperative period followed by clinical control every three months. He also recommended analysing morphology, location, extent and homogeneity of lesions in every clinical examination.

Bhattacharyy [11], conducted a retrospective case control study including 10 patients and concluded that OLP/SCC subjects may exhibit better actuarial survival than SCC patients despite a higher rate of local recurrence and second primary SCC.

OLP described in our patient was classic in its clinical presentation with regard to erosion and chronic inflammation but was characterized by a long evolution period. Diagnosis of squamous cell carcinoma was established at early stage because of clinical and histopathological follow-up. This early diagnosis made possible for the patient to benefit from non-invasive excisional surgery with respect of $5 \mathrm{~mm}$ resection margins. Patient was invited to strict follow-up every 2 months in accordance with Mignogna recommendation to detect local recurrence or second primary carcinoma.

\section{CONCLUSIONS}

The present case supports the view that OLP may undergo malignant transformation, and that this does not require exogenous carcinogens. There is still current uncertainty about the malignant potential of OLP. Microscopic and clinical appearance is still insufficient to differentiate OLP from PVL. This argues for a need to perform routine monitoring of erosive OLP patient especially when having associated other risk factors.

\section{Conflicts of Interest}

The author declares that there is no conflict of interest regarding the publication of this paper.

\section{REFERENCES}

1. Pindborg JJ, Reichart PA, Smith CJ, van der Wall I. Histological typing of cancer and precancer of oral mucosa. 2 nd. New York: Springer. 1997:30.

2. Krutchkoff DJ, Cutler L, Laskowski S. Oral lichen planus: the evidence regarding potential malignant transformation. Oral Pathol. 1978; 7:1-7.

3. Muñoz AA, Haddad RI, Woo SB, Bhattacharyya N. Behavior of oral squamous cell carcinoma in subjects with prior lichen planus. Otolaryngology—Head and Neck Surgery. 2007 Mar;136(3):401-4.

4. Giuliani M, Troiano G, Cordaro M, Corsalini M, Gioco G, Lo Muzio L, Pignatelli P, Lajolo C. Rate of malignant transformation of oral lichen planus: A systematic review. Oral diseases. 2019 Apr;25(3):693-709.

5. Aghbari SM, Abushouk Al, Attia A, Elmaraezy A, Menshawy A, Ahmed MS, Elsaadany BA, Ahmed EM. Malignant transformation of oral lichen planus and oral lichenoid lesions: A meta-analysis of 20095 patient data. Oral oncology. 2017 May 1;68:92-102.

6. $\quad$ van der Waal I, Reichart PA. Oral proliferative verrucous leukoplakia revisited. Oral oncology. 2008;8(44):719-21.

7. Cheng YS, Gould A, Kurago Z, Fantasia J, Muller S. Diagnosis of oral lichen planus: a position paper of the American Academy of Oral and Maxillofacial Pathology. Oral surgery, oral medicine, oral pathology and oral radiology. 2016 Sep 1;122(3):332-54.

8. Liu Y, Messadi DV, Wu H, Hu S. Oral lichen planus is a unique disease model for studying chronic inflammation and oral cancer. Medical hypotheses. 2010 Dec 1;75(6):492-4.

9. Caruntu C, Mitran M, Mitran C, Sarbu I, Rusu LC, Matei C, Constantin C, Neagu M, Georgescu SR. Markers of oral lichen planus malignant transformation. Disease markers. 2018;2018.

10. Mignogna MD, Lo Muzio L, Lo Russo L, Fedele S, Ruoppo E, Bucci E. Oral lichen planus: different clinical features in HCVpositive and HCV-negative patients. International journal of dermatology. 2000 Feb;39(2):134-9.

11. Muñoz AA, Haddad RI, Woo SB, Bhattacharyya N. Behavior of oral squamous cell carcinoma in subjects with prior lichen planus. Otolaryngology—Head and Neck Surgery. 2007 Mar;136(3):401-4. 